\title{
CARACTERIZACIÓN DE UN MODELO DE MEDICIÓN QUE DETERMINE EL NIVEL DE LEGIBILIDAD E INTELIGIBILIDAD EN EL ÁMBITO PEATONAL, VIAL Y CATASTRAL DEL BULEVAR DEL RÍO Y SU PERÍMETRO DE INFLUENCIA
}

\section{Entorno Ciudad}

Ximena Arias Realpe

ximena.arias_realpe@uao.edu.co

Universidad Autónoma de Occidente.

Semillero de investigación en Diseño de Información.

Cali, Valle del Cauca. 


\section{Resumen}

nel mundo existe la necesidad de humanizar los espacios urbanos, empoderar a los ciudadanos

- para que interactúen con los espacios públicos, el patrimonio arquitectónico y las ofertas de servicios turísticos, culturales y oficiales, para buscar suplir sus necesidades y generar cultura cívica y conciencia social. El proyecto de investigación buscó realizar una caracterización de un modelo de medición de la legibilidad (e inteligibilidad) en el ámbito vial, peatonal y catastral del Bulevar del Río y su perímetro de influencia en la ciudad de Santiago de Cali a través de la identificación de las características de una Ciudad Legible, los tipos de usuario existentes en la zona y la estimación de un nivel de legibilidad para el lugar. Por medio de indagación en terreno, consulta de fuentes e interacciones y encuestas con usuarios, la investigación permitió determinar cinco características principales que construyen una ciudad legible, identificar los tipos de usuario existentes en la zona y aproximarse a un nivel cuantitativo de la legibilidad en el Bulevar del Río y su perímetro de influencia. Como resultados se evidenció una ausencia de información suficiente para los peatones en un espacio dispuesto para ellos. La relación entre los ciudadanos y los espacios urbanos está mediada por elementos que permiten su entendimiento para contribuir a la información y toma de decisiones acertadas en el contexto de la vida urbana y de la cultura, para aportar al conocimiento. Al ser el diseño un mediador entre la complejidad urbana y los usuarios, se consideró importante estimar un nivel de legibilidad de la zona para, en el futuro, trabajar en las falencias encontradas y mejorar la experiencia entre los usuarios y la ciudad, para favorecer la apropiación del espacio y el fortalecimiento del tejido social.

\section{Introducción}

Santiago de Cali, actualmente Distrito Especial, Deportivo, Cultural, Turístico, Empresarial y de Servicios, es una ciudad con una enorme oferta cultural, histórica, natural y deportiva, dispuesta a sus residentes y visitantes; pero, parece pasar desapercibida, incluso, para sus propios habitantes.

Al tratarse de un espacio tan importante para la comunidad, el mismo Bulevar del Río es visto como un hito en el imaginario colectivo; aun siendo reciente su construcción, se ha convertido en un punto focal que congrega una cuantiosa población estacionaria y es recorrido por muchas personas con propósitos de entretenimiento o como vía de paso para transeúntes de la zona; pero que, curiosamente, no suele invitar a visitar -o al menos, conocer- los otros hitos y servicios que se encuentran a su alrededor.

Esto último es, en realidad, todo lo contrario a lo que ocurre en una Ciudad Legible, que es aquella que brinda la información suficiente y necesaria para la toma de decisiones, la comprensión de su uso e invita a los usuarios a recorrer y conocer sus espacios e historias. Lo que se traduce en un espacio inclusivo, sostenible y amigable con sus beneficiarios.

Lo mencionado es visto como una oportunidad para caracterizar un modelo de medición de la legibilidad -y, por ende, de inteligibilidad- en el ámbito peatonal, vial y catastral del Bulevar del Río y su perímetro de influencia, al tener como referencia el concepto de Ciudad Legible y los aspectos importantes que la caracterizan: la creación de imagen ambiental eficaz, la visualización 
de la información, la señalización apropiada para orientar, el wayfinding y la territorialización del espacio, los tipos de usuarios que se presentan en el Bulevar del Río y su perímetro de influencia, donde se toma en cuenta la información a la que acceden para ubicarse en el espacio o el nivel de experiencia en dicha zona y, finalmente, estimar un nivel de legibilidad que permita plantear alternativas de mejora que puedan mitigar los vacíos existentes en el lugar y, así, hacer de éste un espacio más resiliente con sus habitantes.

Todo lo anterior, para visionar a Cali como una ciudad más legible e inteligible, al buscar que la misma pueda generar un impacto positivo tanto en habitantes como visitantes, aportando al desarrollo sostenible, a la creación de espacios inclusivos y amigables con los usufructuarios, y a la resignificación del lugar a partir del Diseño de la Comunicación Gráfica, enfocado en las necesidades de las personas y en la funcionalidad de los proyectos, para hacer visible y perceptible la información y, de esta manera, construir conocimiento.

\section{Metodología}

El desarrollo metodológico consideró las siguientes fases:

La primera, la cual consistió en determinar los elementos principales y las características que permiten distinguir a una Ciudad Legible, por lo que esta fase tomó en cuenta la indagación de diferentes autores que se han involucrado en el concepto de Ciudad Legible, a través de cinco subcapítulos, los cuales se definen por: ¿Qué es? ¿Para quién? ¿Cómo se hace? ¿Para qué? y ¿Cómo medirla?

La segunda fase consistió en identificar los tipos de usuarios presentes en el Bulevar del Río y su perímetro de influencia, sus motivaciones y su forma de acceder a la información para ubicarse. Para lograrlo, en esta fase se realizaron encuestas presenciales en la zona, a distintas personas de diferentes edades. También se realizó un proceso de seguimiento a un usuario durante su recorrido entre varios hitos de importancia del Bulevar y su perímetro de influencia -a través de la metodología Walkthrough, implementada por Limerick en el Proyecto Infopolis que se realizó en 1998 y 2000 -.

Para el desarrollo de la metodología Walkthrough, se decidió usar como sujeto de prueba, a un usuario que solía recorrer el Bulevar y su perímetro rara vez y conocía poco sus alrededores. Este usuario tenía como reto ir a seis hitos del Bulevar del Río, algunos serían mencionados por su nombre, otros por su dirección y otros por dirección y nombre. De esta forma, podría evidenciarse qué representaba en mayor medida un reto para dicho usuario, si ubicar una dirección a través de calles y carreras, o encontrar un sitio que por su nombre representaba un hito en su imaginario. El desarrollo de esta metodología reflejó las necesidades del usuario al momento de buscar lugares, y a qué recurría para encontrar información durante el proceso.

La tercera y última fase fue la de estimar un nivel de legibilidad -y por ende, de inteligibilidad-, al tener en cuenta el ámbito vial, peatonal y catastral del Bulevar del Río y su perímetro de influencia, para lo cual se realizó una segunda encuesta, que tenía en cuenta los elementos 
característicos de una Ciudad Legible, adquiridos en la primera etapa, y estaban adaptados al contexto del Bulevar del Río y su perímetro de influencia.

Con los usuarios ya encontrados en la etapa anterior, se estimaría un nivel de legibilidad propio del espacio y adaptado a las necesidades y a la forma de resolverlas de dicho lugar. La segunda encuesta contó con preguntas clasificadas en las distintas características de una Ciudad Legible -sin contar la categoría de Wayfinding, pues éste es un sistema ausente en la ciudad de Cali-, y, de esta forma, los usuarios calificaron de 1 a 5 su percepción con respecto a los diferentes elementos que hacen legible dicho espacio.

\section{Resultados}

El proyecto permitió determinar cinco características principales de la Ciudad Legible, a saber: la creación de imagen ambiental eficaz, la visualización de la información, la señalización apropiada para orientar, el wayfinding y la territorialización del espacio. A su vez, se identificaron los tipos de usuario presentes en el Bulevar del Río; una primera clasificación basada en el nivel de experiencia de los habitantes y visitantes; y, la segunda, clasificada en cuatro niveles, según la información a la que recurren las personas para ubicarse en la zona, como se indica a continuación:

Nivel 1: Aquellas personas que sólo usan hitos (lugares de referencia) para ubicarse.

Nivel 2: Personas que solamente usan direcciones para ubicarse.

Nivel 3: Aquellas personas que usan tanto hitos como direcciones (ya sea calles, carreras o ambas).

Nivel 4: Personas que a pesar de tener los anteriores recursos a su disposición, no se ubican.

Finalmente, se estimó un nivel cuantitativo de legibilidad en la zona, calificando una a una las características que hacen una Ciudad Legible en el contexto del Bulevar del Río, y según la percepción de los usuarios, con los resultados que se muestran a continuación (la calificación de 1 a 5 , se indica en el paréntesis):

Creación de imagen ambiental eficaz (3.1): al ser la característica con el valor más alto, este resultado refleja el nivel de adaptación que han desarrollado los usuarios para encontrar un sentido en el lugar y darle orden e identidad, ayudándose de estímulos visuales como los hitos que hay en la zona. Ya que la imagen ambiental requiere tanto de recursos ofrecidos por el medio ambiente como de la interpretación del usuario, la capacidad de adaptación de las personas en una zona con ausencia de información, resulta bastante importante para lograr una imagen ambiental pertinente, para ubicarse y orientarse, al ser la capacidad de adaptación un elemento vital para sobrevivir a la complejidad del espacio. 
Visualización de la información (2.7): al ser esta la categoría con el resultado más bajo, queda en evidencia la ausencia de información perceptible y clara para el usuario, quien, debido a ello, se ve obligado a recurrir a la tradición oral para llegar a sus destinos -lo que se traduce en preguntarle a la primera persona que se encuentre en el camino, si cuenta con la suerte de que dicha persona sepa realmente cómo llegar o no-.

Señalización apropiada para orientar (2.9): a pesar de que la naturaleza de la señalización está relacionada con el ámbito vial, sin tener en cuenta contextos particulares, y es, prácticamente, regulado a nivel global, este sistema resulta útil en cuanto a la identificación de nomenclatura predial y vial, distinguiendo calles, carreras y el número propio de las casas; sin embargo, y aunque la calificación pareciera más regular que mala, se debe tener en cuenta que este elemento resulta la única información visual y perceptible para el usuario. Pareciera irónico creer que un sistema de señales pensado para el ámbito vial, sea lo único que se ofrece en un espacio $100 \%$ peatonal, y que sus propios usuarios no se sientan del todo satisfechos con dicho sistema.

Wayfinding (0): este es un sistema bastante vital para el peatón, pero que, aun así, no está presente en la ciudad de Cali.

Territorialización del espacio (2.9): aunque el Bulevar del Río es un espacio bastante apreciado por quienes lo frecuentan -tanto habitantes como visitantes de la ciudad-, el hecho de no conocer, en su totalidad, lo que puede encontrar en ese lugar o más allá, genera cierta sensación de inseguridad e insatisfacción, reflejadas en una territorialización del espacio con una calificación regular.

Legibilidad de la zona (2.3): esta calificación baja para la legibilidad de la zona, refleja cierto desinterés por dicho espacio, por parte de los usuarios, al descuidar la información que le brinda y, con ello, se convierte en un lugar mudo que se olvida de invitar a conocer más de él mismo a sus beneficiarios.

Ahora bien, tratándose de un lugar tan importante en la ciudad y contando con una ubicación estratégica que lo conecta con 87 bienes de interés cultural, queda en evidencia que la misma ciudad, en caso de ser medida de la misma forma, puede acabar en un resultado igual o más bajo.

Al comprender que el Diseño Gráfico es funcional y, por ende, busca solucionar problemas en la sociedad (completamente diferente al arte que tiene un carácter subjetivo y de autoexpresión), juega un papel importante en la construcción de una Ciudad Legible, pues el Diseño Gráfico es resultado de las investigaciones y análisis, para garantizar una mejor calidad de vida y, seguramente, belleza en cuanto entiende cómo plasmar visualmente unos resultados prácticos provenientes de las necesidades de los usuarios.

La caracterización mediante indicadores cuantitativos de las variables asociadas a la legibilidad de una ciudad o espacio urbano específico, resultantes de este proyecto, permite diseccionar el fenómeno en componentes específicos, para permitir su abordaje integral 
utilizando, además, el concepto de las tareas visuales, en el cual cada necesidad de información encuentra su respuesta en los sistemas de información dispuestos por profesionales del diseño de información, diseño de wayfinding, urbanistas y planificadores urbanos.

\section{Conclusión}

El saber las falencias, potencialidades y áreas no atendidas en relación a la carencia de elementos que medien la relación entre los usuarios y la ciudad, permite optimizar las estrategias y objetos diseñados que admitan hacer legible la ciudad, al dar respuestas a las necesidades de los usuarios en torno a temas como la orientación, la ubicación y la construcción de tejido social en torno a los discursos culturales, históricos y patrimoniales de los lugares que se habita y en los que se desarrolla la vida, además de generar apropiación e identificación social.

\section{Referencias}

Borthagaray, A. \& Apel-Muller, M. (enero-diciembre, 2014). La ciudad legible: desafío para las ciudades y sus diseñadores. Revista de arquitectura, 16(1). Recuperado de https:// revistadearquitectura.ucatolica.edu.co/issue/view/15

Costa, J. (1987). Señalética. Barcelona: Ediciones CEAC, S.A.

Dürsteler, J. C. (2003). Visualización de la información. Una visita guiada. Barcelona: Gestión 2000.

Gibson, D. (2009). The Wayfinding Handbook. Information design for public places. New York: Princeton Architectural Press.

Golledge, R. G. (1999). Wayfinding Behavior: cognitive mapping and other spatial processes. Baltimore: The Johns Hopkins University Press.

Grupo de Investigación Nación, Cultura, Memoria. Universidad del Valle. (2012). Historia de Cali Siglo XX. Santiago de Cali: Programa Editorial Facultad de Humanidades, Universidad del Valle.

Hernández Sampieri, R.; Fernández Collado, C. \& Baptista Lucio, P. (2014). Metodología de la investigación. México D.F.: Mcgraw-Hill Interamericana.

Proyecto Infopolis. (1998/2000). Metodología Walkthrough. Limerick. Recuperado de http://www.infopolis.es/web/infopolis.html

Secretaría de Turismo de Cali. (s.f.). Cali tiene cosas que contarte. Santiago de Cali, Colombia: Alcaldía del Municipio de Cali

Secretaría de Turismo de Cali. (22 de febrero de 2018). Alcaldía de Santiago de Cali [Mensaje en sitio web]. Recuperado de http://www.cali.gov.co/turismo/publicaciones/139216/ cali-baila/

Silva, A. (2006). Imaginarios urbanos. Bogotá, DC.: Editorial Nomos.

Taylor, S., \& Bodgan, R. (1987). Introducción a los métodos cualitativos de investigación. Buenos Aires: Paidós.

Uribe Orozco, M. F. (2016). Diseño de información. Una herramienta para el uso y apropiación del transporte público. Santiago de Cali: Universidad Autónoma de Occidente. 